\title{
Novel Features of Nuclear Forces and Shell Evolution in Exotic Nuclei
}

\author{
Takaharu Otsuka, ${ }^{1,2}$ Toshio Suzuki, ${ }^{3}$ Michio Honma, ${ }^{4}$ Yutaka Utsuno, ${ }^{5}$ Naofumi Tsunoda, ${ }^{1}$ \\ Koshiroh Tsukiyama, ${ }^{1}$ and Morten Hjorth-Jensen ${ }^{6}$ \\ ${ }^{1}$ Department of Physics, University of Tokyo, Hongo, Bunkyo-ku, Tokyo 113-0033, Japan \\ ${ }^{2}$ Center for Nuclear Study, University of Tokyo, Hongo, Bunkyo-ku, Tokyo 113-0033, Japan \\ ${ }^{3}$ Department of Physics, Nihon University, Sakurajosui, Setagaya-ku, Tokyo 156-8550, Japan \\ ${ }^{4}$ Center for Mathematical Sciences, University of Aizu, Tsuruga, Ikki-machi, Aizu-Wakamatsu, Fukushima 965-8580, Japan \\ ${ }^{5}$ Japan Atomic Energy Agency, Tokai, Ibaraki, 319-1195 Japan \\ ${ }^{6}$ Department of Physics and Center of Mathematics for Applications, University of Oslo, N-0316 Oslo, Norway
}

(Received 29 September 2009; published 4 January 2010)

Novel simple properties of the monopole component of effective nucleon-nucleon interactions are presented, leading to the so-called monopole-based universal interaction. Shell structures are shown to change as functions of $N$ and $Z$, consistent with experiments. Some key cases of this shell evolution are discussed, clarifying the effects of central and tensor forces. The validity of the present tensor force is examined in terms of the low-momentum interaction $V_{\text {lowk }}$ and the $Q_{\text {box }}$ formalism.

Exotic nuclei provide us with new phenomena not found in stable nuclei. One of them is the evolution of shell structure as a function of the proton number $(Z)$ or neutron number $(N)$ [1]. The evolution ends up, in some cases, with the appearance of new magic numbers and/or the disappearance of conventional ones. As $Z$ increases, there are more exotic isotopes between the $\beta$-stability line and the drip line, creating a wider frontier. Most of such exotic nuclei are far inside the drip line, being well bound. The driving force behind the change in their structure should be the combination of the unbalanced $Z / N$ ratio and the nuclear force [2]. Thus, it is crucial to see robust basic features of the nuclear force in exotic nuclei. We present in this Letter novel simple properties of the monopole component of shell-model interactions which can reproduce experimental data. While the shell evolution due to the tensor force has been suggested in [3], we introduce here an interaction which includes the central force also, moving closer to the complete picture.

We start with selected shell-model nucleon-nucleon $(N N)$ interactions which are successful in describing experimental data. These interactions were obtained based on so-called microscopic interactions, derived, for example, with the $G$-matrix approach [4,5] starting from a bare $N N$ interaction and incorporating short-range repulsion and core polarization. In order to reproduce experimental data, however, the microscopic interaction has to be modified empirically, as is the case for the families of the USD [6], KB3 [7] and GXPF1 [8] interactions. We shall take $p f$-shell first, and analyze the GXPF1A interaction [9] and the corresponding $G$-matrix interaction [5].

The monopole matrix element of a given two-body interaction $V$ is defined as

$$
v_{m ; j, j^{\prime}}=\sum_{k, k^{\prime}}\left\langle j k j^{\prime} k^{\prime}|V| j k j^{\prime} k^{\prime}\right\rangle / \sum_{k, k^{\prime}} 1,
$$

where $j$ denotes a single-particle orbit with $k$ being its magnetic substate and $\langle\cdots|V| \cdots\rangle$ is the antisymmetrized two-body matrix element. The monopole component of $V$ is written, for $j \neq j^{\prime}$, as $\sum_{j, j^{\prime}} v_{m ; j, j^{\prime}} n_{j} n_{j^{\prime}}$, where $n_{j}$ is the occupation number (operator) of orbit $j$ [10]. The monopole component is nothing but the average over all orientations. It was introduced by Bansal and French [11], while its relevance to the effective shell-model interaction was discussed by Poves and Zuker [7]. Recently, the monopole component of the spin-isospin interaction has been shown to modify even the magic structure in exotic nuclei [2], and the specific and substantial role of the tensor force was shown in Ref. [3]. Note that $v_{m ; j, j^{\prime}}$ is defined either with isospin, $T=0$ or 1 , or in the proton-neutron scheme, while " $j, j^{\prime \prime}$ " may be omitted for brevity.

The importance of the monopole interaction for exotic nuclei originates in its linearity. As the orbit $j^{\prime}$ is occupied, the single-particle energy (SPE) of an orbit $j, \epsilon_{j}$, is changed by [10],

$$
\Delta \epsilon_{j}=v_{m ; j, j^{\prime}} n_{j^{\prime}}
$$

For $j^{\prime}=g_{9 / 2}$ as an example, $n_{j^{\prime}}$ takes values up to 10 . Thus, the effect of the monopole component can be magnified considerably. By moving along the nuclear chart, one can indeed change a particular $n_{j^{\prime}}$ substantially. This highlights the physics of exotic nuclei compared to that of stable nuclei, and it is of keen and urgent interest to clarify general and robust features of the monopole interaction. At shell closures, the monopole component produces effects according to Eq. (2), whereas effects of other multipole components vanish. The monopole component governs (spherical) SPEs on top of closed (sub)shells. In open shell systems, its effects can be viewed through Eq. (2) as effective SPEs. As the surface deformation with low exci- 
tation energies is a Jahn-Teller effect, the SPEs are crucial for collectivity too.

Figure 1(a) shows $v_{m ; j, j^{\prime}}$ for isospin $T=0$ from the GXPF1A interaction, the $G$-matrix interaction [5] and the tensor force in the $p f$ shell. The tensor force refers, in all parts of Fig. 1, to the $\pi+\rho$ meson exchange force used in [3]. The orbits $\left(j, j^{\prime}\right)$ are grouped as $(f, f),(p, p)$, and $(f, p)$. In Fig. 1(a), we find two distinct kinks in the tensorforce values for the $(f, f)$ and the $(p, p)$ groups, and the same kinks appear also in the GXPF1A and the $G$-matrix results. Note that each kink is a consequence of the general rule suggested in [3]. The similarities are remarkable. To shed more light on this, in Fig. 1(b) we subtract the tensorforce contribution from the GXPF1A and the $G$-matrix values. This results in almost flat curves. The $(f, f)$ and $(p, p)$ cases show almost the same values, while the $(f, p)$ shows higher but still nearly flat values. This can be understood in terms of radial integral of the central force: in the former case the radial wave functions are the same between $j$ and $j^{\prime}$, while they are different in the latter. The flatness suggests a longer-range central force. In order to incorporate these features, we introduce a central Gaussian interaction as

$$
V_{c}=\sum_{S, T} f_{S, T} P_{S, T} \exp \left(-(r / \mu)^{2}\right)
$$

where $S(T)$ means spin (isospin), $P$ denotes the projection operator onto the channels $(S, T)$ with strength $f$, and $r$ and $\mu$ are the internucleon distance and Gaussian parameter, respectively. Figure 1(b) shows results obtained by $f_{0,0}=$ $f_{1,0}=-166 \mathrm{MeV}$ and $\mu=1.0 \mathrm{fm}$. The agreement with GXPF1A is remarkable, considering the simplicity of the model. Thus, we can describe the monopole component by two simple terms: the tensor force generates "local" variations, while the Gaussian central force produces a flat "global" contribution. It is worth mentioning that $\mu=$ $1.0 \mathrm{fm}$ is reasonable from the viewpoint of $N N$ interaction, and deviations from it, including the zero-range limit, worsen the agreement.

Figure 1(c) shows $v_{m}$ 's for $T=1$. They are grouped for pairs of $j=j^{\prime}$ and the rest. The former corresponds to the standard BCS-type pairing cases. We first stress that the basic scale is quite different between $T=0$ and 1: $v_{m}$ 's of GXPF1A are in the range -2.5 to $-1 \mathrm{MeV}$ for $T=0$, whereas for $T=1$ they are in the range -0.3 to $+0.2 \mathrm{MeV}$. The sharp rise for $j=j^{\prime}=p_{1 / 2}$ occurs in all three interactions as a characteristic fingerprint of the
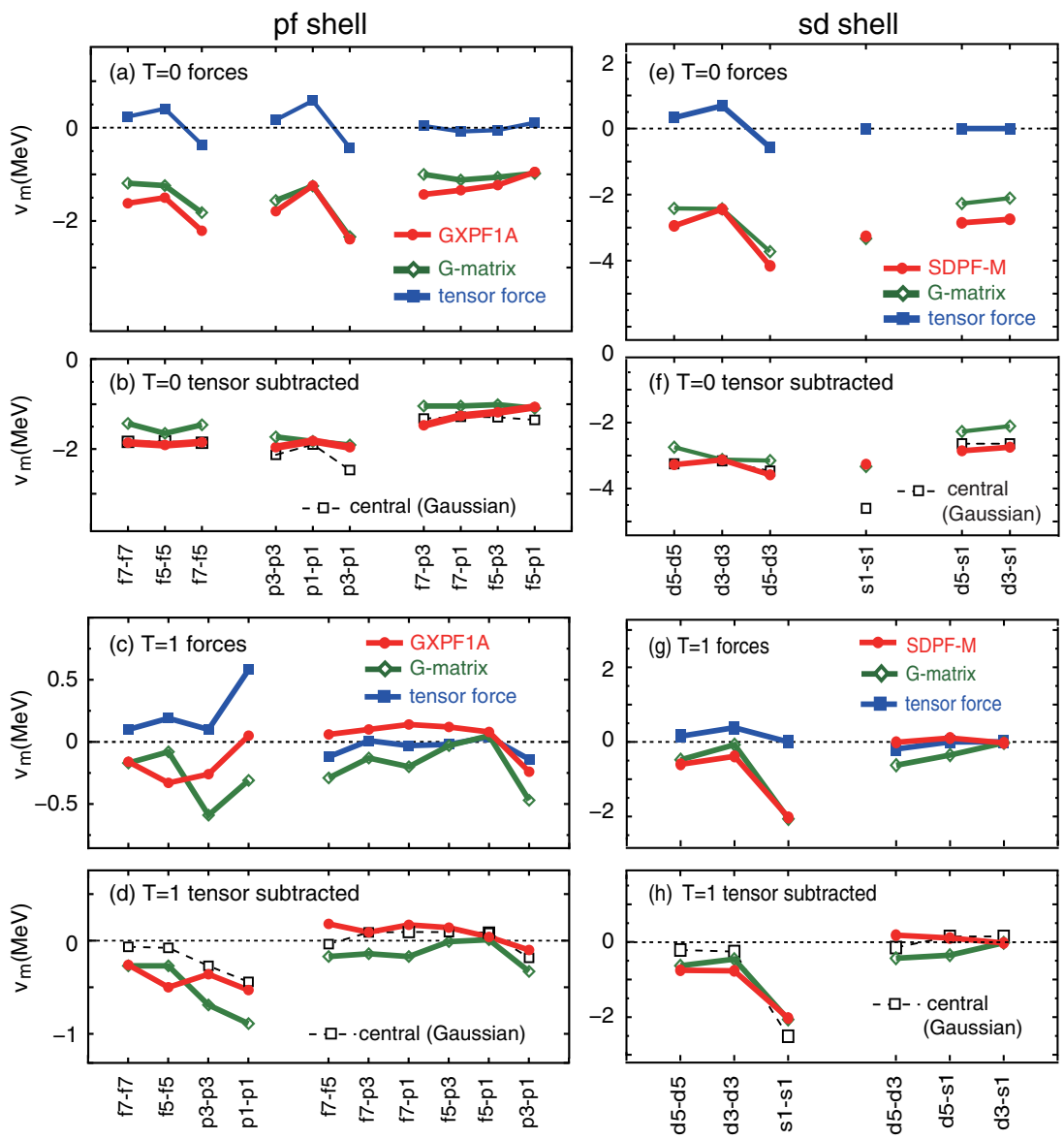

FIG. 1 (color online). Monopole matrix elements of various forces for (a)-(d) $p f$ and (e)-(h) $s d$ shells. In (b),(d),(f),(h), the tensorforce effect is subtracted from the others, and results from a Gaussian central force are shown. 
(a) central force : Gaussian (strongly renormalized)

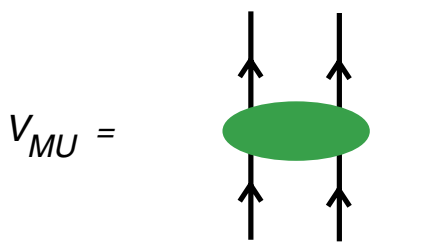

(b) tensor force: $\pi+\rho$ meson exchange

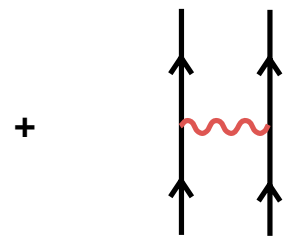

FIG. 2 (color online). Diagrams for the $V_{\mathrm{MU}}$ interaction.

tensor force. Note that $v_{m}$ 's for the GXPF1A interaction ( $G$ matrix) are mostly repulsive (attractive) for $j \neq j^{\prime}$. We subtract the tensor contribution as was done in Fig. 1(b), and show the result in Fig. 1(d) as well as those of the Gaussian central force with $f_{0,1}=0.6 f_{0,0}$ and $f_{1,1}=$ $-0.8 f_{0,0}$. The basic feature can be reproduced, while $T=$ $1 j=j^{\prime}$ cases need a certain attention as they show some deviations also in $s d$ shell as shown below.

Figures 1(e)-1(h) exhibit $v_{m}$ 's in the $s d$ shell, similar to what is shown in Figs. 1(a)-1(d). The SDPF-M interaction [12] is taken as the realistic interaction. All features discussed for the $p f$ shell are seen, and the tensor-subtracted values are reproduced by the same Gaussian central force. One sees repulsive corrections to $v_{m}$ 's from the $G$ matrix for $T=1$ and $j \neq j^{\prime}$, similar to our findings in the $p f$ shell. This correction is linked with the oxygen drip line, its origin has been a puzzle, but has recently been resolved [13].

Based on the above results, we introduce the monopolebased universal interaction, $V_{\mathrm{MU}}$. As shown in Fig. 2, $V_{\mathrm{MU}}$ consists of two terms. The first term is the Gaussian central force discussed so far, and should contain many complicated processes including multiple meson exchanges. The second one is the tensor force comprised of $\pi$ and $\rho$ meson exchanges [3]. The $V_{\mathrm{MU}}$ interaction resembles Weinberg's original model for chiral perturbation theory [14], if one replaces Fig. 2(a) by contact terms and 2(b) by the one- $\pi$ exchange potential.

Figure 3 shows applications of $V_{\mathrm{MU}}$, with the parameters fixed above, to the shell evolution assuming a filling configuration. Figure 3(a) depicts neutron SPEs around $N=$ 20 for $Z=8-20$. Starting from SDPF-M SPEs at $Z=8$, one sees the evolution of the $N=20$ gap, in a basically consistent manner with other shell-model studies $[12,15]$. While the change is monotonic without the tensor force, the tensor force produces a sharp widening from $Z=8$ to 14 , and then stabilizes the gap towards $Z=20$. It is worth mentioning that the normal SPEs arise at $Z=20$, whereas at $Z=8$ the inversion between $f_{7 / 2}$ and $p_{3 / 2}$ occurs and $d_{3 / 2}$ is rather close to $p_{3 / 2}$, leaving the major gap at $N=$ 16. The central force lowers the neutron $d_{3 / 2}$ SPE more than the $f_{7 / 2}$ SPE as protons occupy the $s d$ shell due to larger overlaps, yielding a wide $N=20$ gap at ${ }^{40} \mathrm{Ca}$. The $N=20$ gap at $Z \sim 14$ is, however, largely due to the tensor force, and becomes smaller if protons are excited to $d_{3 / 2}$.

Figure 3(b) shows proton SPEs for the $Z=28$ core of ${ }^{68-78} \mathrm{Ni}$, by starting from empirical values [16] at $N=40$. The SPE of $p_{1 / 2}$ is not known empirically, and is placed above $p_{3 / 2}$ by the energy difference predicted by the GXPF1A interaction. The orbit $f_{5 / 2}$ crosses $p_{3 / 2}$ at $N=$ 45 consistently with a recent experiment [17], and the $f_{7 / 2}-f_{5 / 2}$ splitting is reduced by $2 \mathrm{MeV}$ from $N=40$ to 50 . For both, the tensor force plays crucial roles. This lowering of $f_{5 / 2}$ is seen in other shell-model results, while the change is about a half of the present value [18].

Figure 3(c) shows neutron SPEs relative to $d_{5 / 2}$ on top of ${ }^{90} \mathrm{Zr}-{ }^{100} \mathrm{Sn}$, starting from empirical values at $Z=40 \mathrm{ob}-$ tained by averaging with spectroscopic factors [19]. The lowering of $g_{7 / 2}$ is remarkable [20]. If there were no tensorforce effects, $g_{7 / 2}$ and $h_{11 / 2}$ do not repel, ending up with quite a different shell structure for ${ }^{100} \mathrm{Sn}$, making this nucleus much softer. The closer spacing of $g_{7 / 2}$ and $d_{5 / 2}$ in ${ }^{101} \mathrm{Sn}$ seems to be seen experimentally [21].
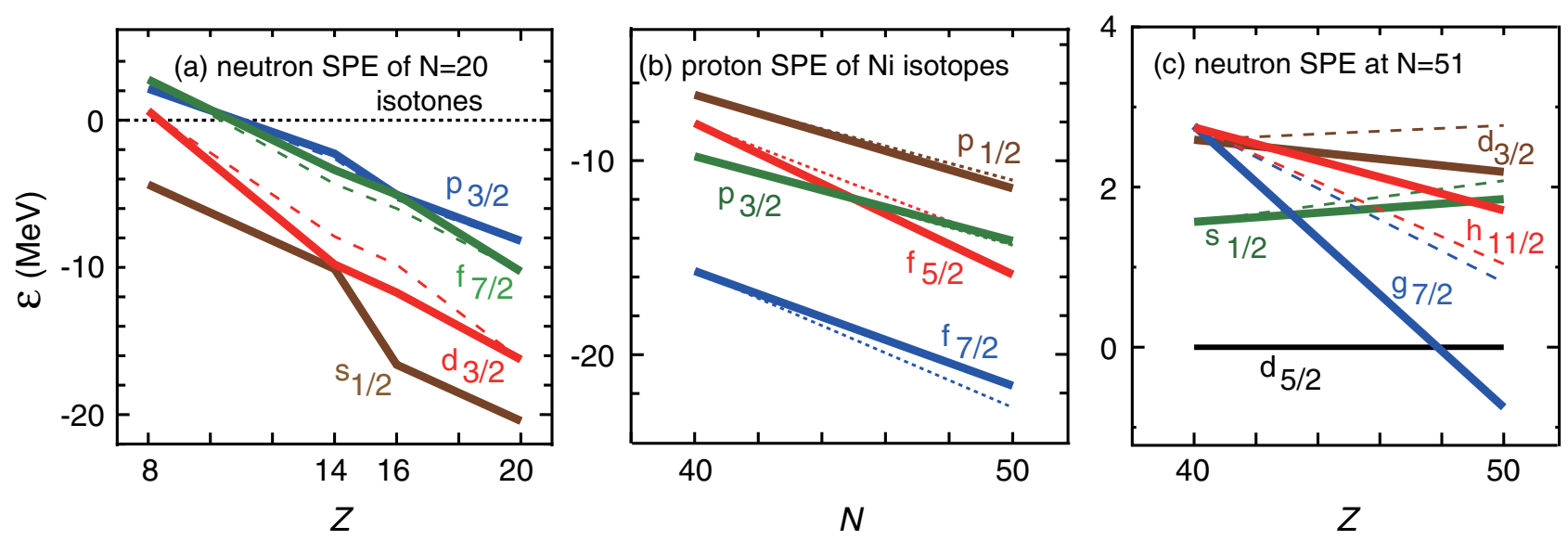

FIG. 3 (color online). Single-particle energies calculated by $V_{\mathrm{MU}}$ interaction. The dashed lines are obtained by the central force only, while the solid lines include both the central force and the tensor force. Some states, e.g. $f_{7 / 2}$ in (b), are hole states. 

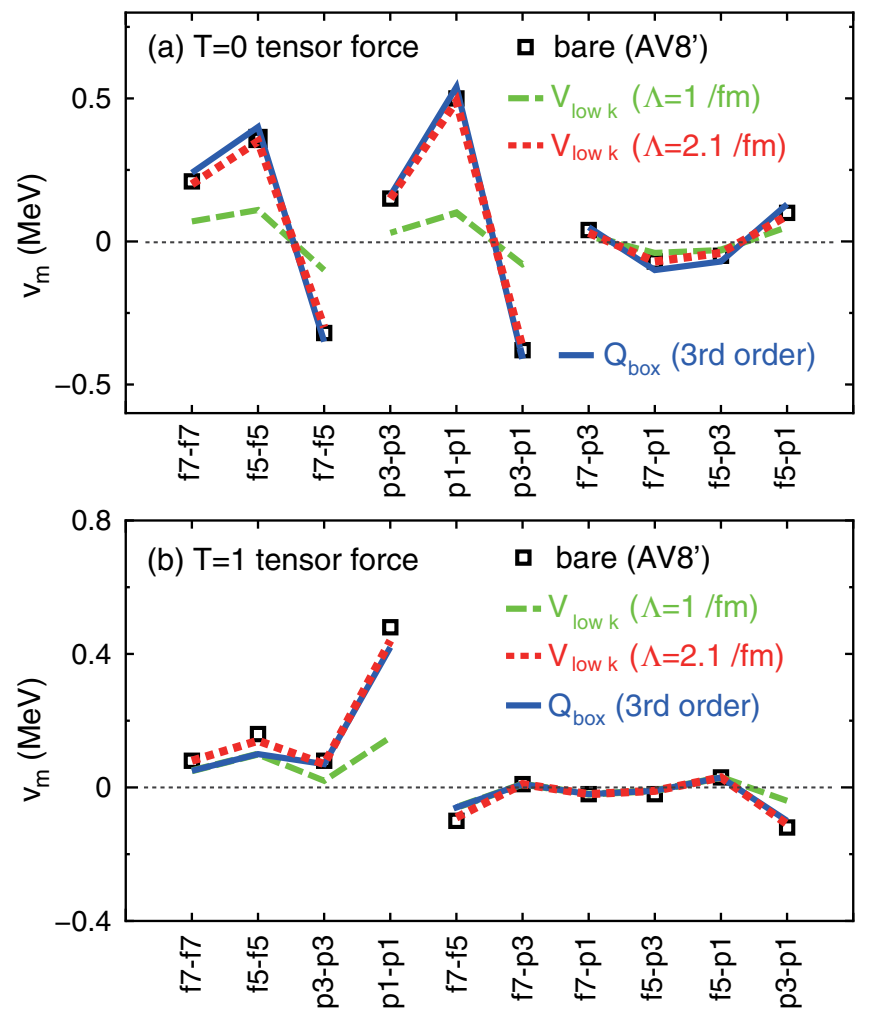

FIG. 4 (color online). Tensor forces in AV8' interacton, in lowmomentum interactions obtained from AV8', and in the thirdorder $Q_{\text {box }}$ interaction for (a) $T=0$ and (b) $T=1$.

We note that the proton $g_{7 / 2}-h_{11 / 2}$ spacing in $\mathrm{Sb}$ isotopes becomes wider by $\sim 2 \mathrm{MeV}$ in going from $N=$ 64 to 82 in agreement with experiment [22].

We now discuss whether or not the simple tensor force in $V_{\mathrm{MU}}$ can be explained microscopically. We take the AV8' interaction [23] and examine how the tensor force obtained by the spin-tensor decomposition changes in the following processes. We derive a low-momentum interaction $V_{\text {lowk }}$ [24] and calculate $v_{m}$ 's, as shown in Figs. 4(a) and 4(b) for $T=0,1$, varying the cutoff parameter $\Lambda$. For the usual value $\Lambda=2.1\left(\mathrm{fm}^{-1}\right)$, the result is very close to the bare AV8' tensor-force contribution. We then perform the thirdorder $Q$-box calculation with folded diagram corrections [5], in order to include medium effects like core polarization. The result still resembles $v_{m}$ 's of the bare tensor part. Thus we can confirm that the treatments of the short-range correlation and the medium effects do not change much $v_{m}$ 's of the tensor force. This near independence may be interpreted in terms of specific and complicated angular momentum coupling in the tensor force. For instance, the second-order perturbation by two tensor forces yield mainly a central force. For unusual values like $\Lambda=$ $1\left(\mathrm{fm}^{-1}\right)$, deviations arise, as expected.

The central force depends strongly on $\Lambda$. For $\Lambda=$ $2.1\left(\mathrm{fm}^{-1}\right), v_{m}$ 's of the central part of $V_{\text {lowk }}$ are scattered around the values of Fig. 1(b). This result is promising, but more studies are needed.

In summary, we have presented novel general properties of the monopole interactions, and introduced the $V_{\mathrm{MU}}$ interaction consisting of simple central and tensor forces. The persistency of the bare tensor force is examined by the $V_{\text {lowk }}$ and $Q_{\text {box }}$ formalisms. The $V_{\mathrm{MU}}$ produces a variety of the shell evolution, connecting stable and exotic nuclei, e.g., exotic $\mathrm{Ne}-\mathrm{Mg}$ with ${ }^{40} \mathrm{Ca},{ }^{68} \mathrm{Ni}$ with exotic ${ }^{78} \mathrm{Ni}$, and ${ }^{90} \mathrm{Zr}$ with exotic ${ }^{100} \mathrm{Sn}$. The shell structure appears to vary considerably in exotic nuclei. As $V_{\mathrm{MU}}$ has been introduced based on monopole properties, tests of its validity by full shell-model calculations and possible refinements including multipole components are of great interest, as well as more predictions by $V_{\mathrm{MU}}$.

We thank Dr. H. Grawe for valuable discussions. This work was supported in part by a Grant-in-Aid for Specially Promoted Research (13002001). This work has been supported by the JSPS Core-to-Core Program EFES, and is a part of the RIKEN-CNS joint research project on largescale nuclear-structure calculations.

[1] A. Gade and T. Glasmacher, Prog. Part. Nucl. Phys. 60, 161 (2008); O. Sorlin and M.-G. Porquet, Prog. Part. Nucl. Phys. 61, 602 (2008).

[2] T. Otsuka et al., Phys. Rev. Lett. 87, 082502 (2001).

[3] T. Otsuka et al., Phys. Rev. Lett. 95, 232502 (2005).

[4] T. T.S. Kuo and G.E. Brown, Nucl. Phys. A114, 241 (1968).

[5] M. Hjorth-Jensen, T. T. S. Kuo and E. Osnes, Phys. Rep. 261, 125 (1995).

[6] B. A. Brown and B. H. Wildenthal, Annu. Rev. Nucl. Part. Sci. 38, 29 (1988).

[7] A. Poves and A. Zuker, Phys. Rep. 70, 235 (1981).

[8] M. Honma et al., Phys. Rev. C 65, 061301(R) (2002); Phys. Rev. C 69, 034335 (2004).

[9] M. Honma et al., Eur. Phys. J. A 25, Suppl. 1, 499 (2005).

[10] For $j=j^{\prime}$ for identical nucleons, form differs slightly.

[11] R. K. Bansal and J. B. French, Phys. Lett. 11, 145 (1964).

[12] Y. Utsuno et al., Phys. Rev. C 60, 054315 (1999).

[13] T. Otsuka et al., arXiv:0908.2607.

[14] S. Weinberg, Phys. Lett. B 251, 288 (1990).

[15] F. Nowacki and A. Poves, Phys. Rev. C 79, 014310 (2009).

[16] H. Grawe et al., Eur. Phys. J. A 25, Suppl. 1, 357 (2005).

[17] K. T. Flanagan et al., Phys. Rev. Lett. 103, 142501 (2009).

[18] J. Duflo and A. P. Zuker, Phys. Rev. C 59, R2347 (1999); N. A. Smirnova et al., Phys. Rev. C 69, 044306 (2004).

[19] http://www.nndc.bnl.gov/ensdf/

[20] P. Federman and S. Pittel, Phys. Lett. B 69, 385 (1977).

[21] D. Seweryniak et al., Phys. Rev. Lett. 99, 022504 (2007); R. Gryzywacz et al. (private communication).

[22] J. P. Schiffer et al., Phys. Rev. Lett. 92, 162501 (2004).

[23] B. S. Pudliner et al., Phys. Rev. C 56, 1720 (1997).

[24] S. K. Bogner, T. T. S. Kuo, and A. Schwenk, Phys. Rep. 386, 1 (2003). 